\title{
Cent ans de signalisation latérale en Europe. Divergences et convergences
}

Daniel Wurmser

\section{CpenEdition}

\section{Journals}

Édition électronique

URL : https://journals.openedition.org/rhcf/1976

DOI : 10.4000/rhcf.1976

\section{Éditeur}

Rails \& histoire

\section{Édition imprimée}

Date de publication : 1 février 2003

Pagination : 160-188

ISBN : 0996-9403

ISSN : 0996-9403

\section{Référence électronique}

Daniel Wurmser, «Cent ans de signalisation latérale en Europe. Divergences et convergences », Revue d'histoire des chemins de fer [En ligne], 26 | 2003, mis en ligne le 21 janvier 2015, consulté le 22 avril 2022. URL : http://journals.openedition.org/rhcf/1976 ; DOI : https://doi.org/10.4000/rhcf.1976 


\section{Daniel WURMSER}

\section{Cent ans de signalisation latérale en Europe Divergences et convergences}

On sait depuis longtemps que le chemin de fer a besoin de signaux d'arrêt précédés de signaux d'avertissement qui mettent en mesure de les respecter. Mais la signalisation ne s'est pas réduite à cela, car des perfectionnements incessants, la complexité croissante des installations et des besoins nouveaux allaient lui donner sa physionomie actuelle. Couvrant une période qui s'étend, en gros, de 1895 à 1995, cette étude résume les facteurs qui ont le plus marqué ce domaine essentiel de la sécurité ferroviaire, sans oublier que, si des démarches similaires ont été suivies un peu partout en Europe, elles n'ont été nulle part simultanées. De plus, la période considérée permet, à son origine, d'éliminer les balbutiements des débuts pour ne retenir que les pratiques durables et, à sa fin, de constater que la signalisation latérale est désormais menacée par de nouvelles technologies.

Les facteurs marquants peuvent être classés en trois groupes (planche I) :

- Les progrès des installations de sécurité où chacun, sur la base de l'expérience acquise et du progrès technologique (par exemple le recours de plus en plus développé à l'électricité), a amélioré sa signalisation et comblé les lacunes des programmes ou des principes antérieurs;

- les besoins nouveaux qui, sans être directement liés à la signalisation, l'ont influencée (par exemple l'apparition d'aiguilles longues admettant des vitesses en déviation plus élevées) ;

- les influences, variées, extérieures telles que proximité géographique, constructeurs d'équipements, autorités gouvernementales, conflits ou dominations, et l'inévitable obsolescence.

Si les deux premiers facteurs ont favorisé les divergences, le dernier a plutôt joué en sens inverse.

\section{Les progrès des installations de sécurité}

Le premier est le verrouillage des aiguilles, car on ne pouvait franchir les bifurcations qu'à vitesse réduite, quelle que soit la direction donnée, directe ou déviée. Ce perfectionnement a permis de franchir sans ralentir la branche directe des aiguilles. La signalisation a donc dû 


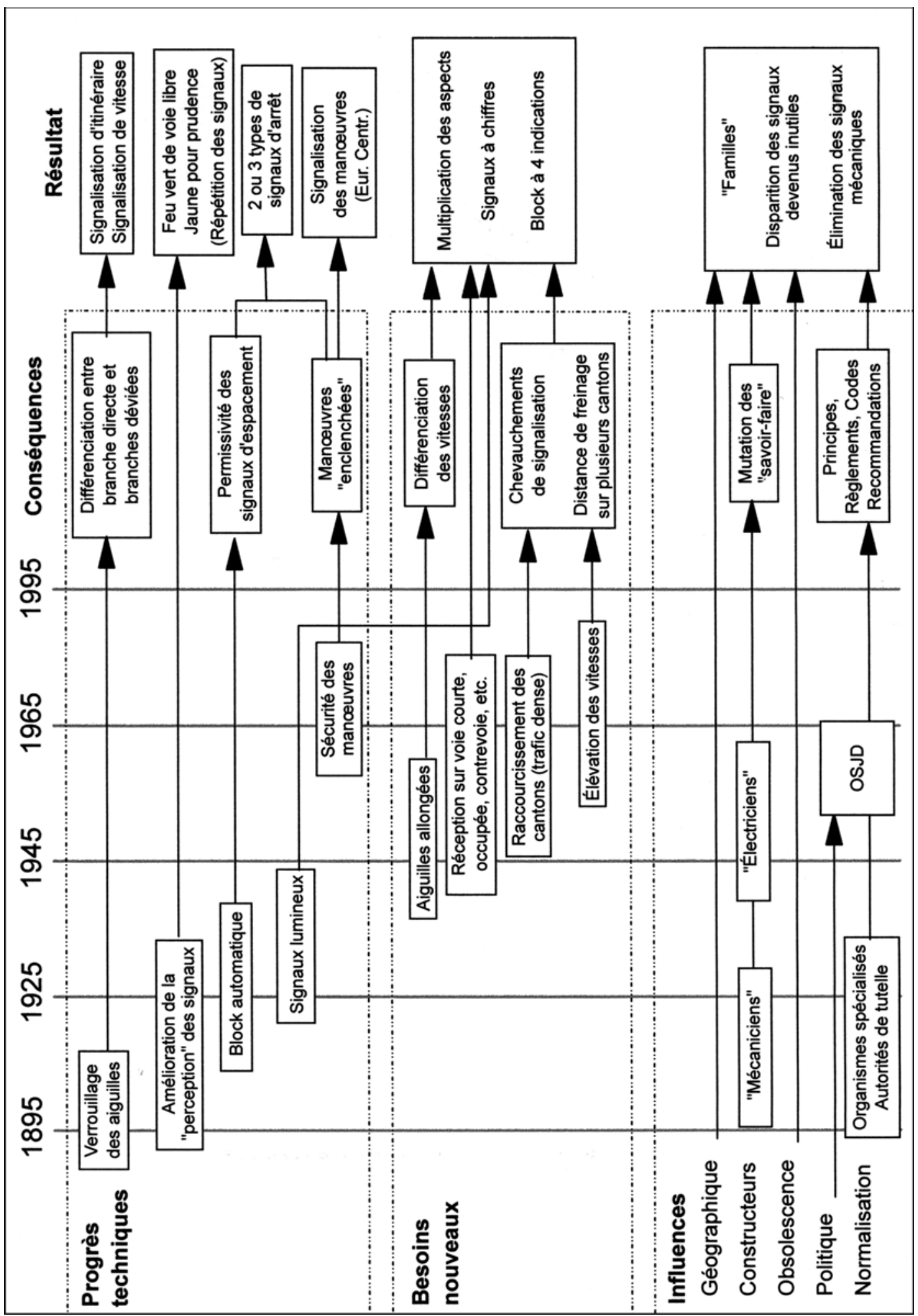


différencier les aspects présentés selon que la branche déviée ou directe est préparée. Dès 1890 elle l'avait fait de deux manières :

- la signalisation d'itinéraire qui n'indique que la direction donnée; il incombe au mécanicien de savoir la vitesse admise par sa connaissance de la ligne ou par le livret de marche de son train ;

- la signalisation de vitesse qui indique s'il faut ralentir ou non et à quelle vitesse ; l'indication de direction vient en second lieu, s'il y a doute sur la direction donnée.

L'amélioration de la perception des signanx et de leur interprétation qui recouvre, entre autres :

- l'adoption du feu vert à la place du feu blanc de voie libre qui évite les conséquences du bris d'un verre coloré et les confusions avec les feux blancs environnants ;

- le choix de la couleur jaune pour les signaux de ralentissement ou de marche prudente ;

- le clignotement des feux qui a permis de créer de nouveaux aspects aisément repérables; les réseaux scandinaves en ont usé dès 1920 pour économiser l'acétylène comprimé qui avait succédé au pétrole pour éclairer les signaux d'avertissement qui sont situés loin des postes ;

- la création de nouveaux aspects pour les signaux d'avertissement pour indiquer sans ambiguité la position du signal annoncé et éviter qu'un mécanicien trouve inopinément une indication plus restrictive que celle qu'il a l'habitude de rencontrer;

- la répétition en cabine des signaux, bien que déjà ancienne, sort du cadre de l'exposé.

Le block automatique, venu des États-Unis dès les années 1900, pose le problème de la conduite à tenir par le mécanicien face à un signal mis à l'arrêt par la condition d'espacement, sans qu'aucun agent ne puisse le renseigner sur place; il en est résulté la permissivité de certains signaux et, donc, un aspect ou un repérage différents de ceux du signal d'arrêt absolu.

L'essor des signaux lumineux à partir de 1920, conséquence du développement des réseaux de distribution d'électricité. Leurs avantages sont tels qu'ils supplanteront progressivement les signaux mécaniques; à l'heure actuelle, ils les ont éliminés de certains pays, principalement dans le nord de l'Europe. Après avoir reconduit les fonctions et les aspects nocturnes de leurs prédécesseurs, ils s'en sont progressivement affranchis ; ils ont surtout facilité la création des aspects relatifs à des 
besoins nouveaux comme la signalisation des limitations de vitesse sur les aiguilles en déviation normales ou allongées, ou comme le block à quatre indications.

L'amélioration de la sécurité des mancuures. Ce problème a surtout concerné les réseaux d'Europe centrale où ce type de mouvements avait fait l'objet de programmes de sécurité simplifiés. Le droit accordé aux manœuvres de franchir fermés les « signaux principaux », qui sont d'arrêt absolu pour les trains, revenait à se priver des garanties offertes par les enclenchements et l'immobilisation des aiguilles (régime de manœuvres dit à « aiguilles libres»). Ce régime a été remis en cause après la Seconde Guerre mondiale avec le développement des postes d'aiguillage " tout-relais ", où les manœuvres relèvent de parcours enclenchés, comme les trains.

\section{Les besoins nouveaux}

Apparus dans l'entre-deux-guerres, ces besoins n’ont cessé de s'amplifier.

Les aiguilles allongées. Leur angle de déviation de plus en plus faible autorise des vitesses de $60,80,100,120 \mathrm{~km} / \mathrm{h}$ ou plus par la branche déviée. L'écart avec les anciens taux de 30 ou $40 \mathrm{~km} / \mathrm{h}$ est donc assez important pour justifier la différenciation des indications. De nouveaux aspects, ou mieux, des signaux à chiffres ajustables au taux voulu ont fait leur apparition avec une version " exécution » et une version « à distance ». Ce fut une autre occasion de diversité.

Le raccourcissement des cantons. Près des grandes gares ou sur des troncs communs chargés, le faible intervalle séparant les trains ou le faible écart entre les postes d'aiguillage (dont les signaux participent souvent au cantonnement) ont conduit à créer des cantons dont la longueur est inférieure aux distances de freinage. Il en est résulté des chevauchements de signalisation, la distance de freinage se répartissant sur deux cantons et, au total, un block à quatre indications au lieu de trois, apte à présenter les signaux d'avertissement, de préavertissement ou répétiteur d'avertissement.

L'élévation des vitesses en pleine voie à partir des années $1960 \mathrm{a} \mathrm{eu}$ un effet similaire, puisque les distances de freinage ont dépassé la longueur des cantons existants qui était d'environ 1500 mètres en block automatique. Une signalisation d'espacement à quatre indications adaptée aux grandes vitesses a été mise en place (par exemple en France avec le feu vert clignotant de préannonce à la place du feu jaune clignotant sur les lignes où la vitesse dépasse $160 \mathrm{~km} / \mathrm{h}$ ). 
Les facilités d'exploitation telles que les réceptions sur voie courte, occupée, en impasse, ou les circulations à contre-voie ont favorisé la multiplication des signaux. En même temps la recherche d'une meilleure souplesse d'exploitation a fait évoluer les rapports qui existaient entre signaux et livret de marche des trains. Ce dernier introduisait en effet trop de rigidité, en indiquant par exemple pour un train sa voie de réception en gare, les vitesses à respecter et l'aspect des signaux, rendant impossible un changement inopiné sans provoquer un arrêt pour non conformité à l'itinéraire attendu. Pour éviter de tels arrêts on a fait donner plus de renseignements aux signaux (et créé de nouveaux aspects) et moins au livret de marche.

\section{Les influences extérieures}

Les influences géographiques sont fréquentes, la signalisation d'un pays influençant celle de ses voisins ; c'est aussi le fait de constructeurs d'équipements qui ont étendu leur zone d'action autour de leur pays d'origine. Le phénomène est net en Europe centrale avec les constructeurs allemands.

Les constructeurs ont souvent proposé les équipements « clés en mains ", des leviers et de la table d'enclenchements aux signaux, en passant par les organes intermédiaires. En signalisation mécanique, les plus notables ont été les constructeurs anglais (Saxby et Farmer, Stevens, Tyer) et allemands (Bruchsal, Jüdel, Siemens) qui tendaient naturellement à exporter les techniques de leur pays d'origine. La France a largement fait appel à la firme Saxby pour les postes d’aiguillage bien que sa signalisation n'obéisse pas au modèle anglais ; curieusement cette firme est ensuite devenue purement française. Le développement de la signalisation lumineuse, du block automatique et des postes « à pouvoir » dans les années vingt ont fait émerger de nouveaux acteurs (dont des sociétés américaines ou leurs filiales). La signalisation devenait un autre métier, où le savoir-faire mécanicien cédait la place au savoir-faire électricien chez les constructeurs comme dans les bureaux d'études des chemins de fer.

Les influences politiques. Elles reflètent l'histoire, les appartenances, les dominations et les conflits. La signalisation anglaise est très présente dans les anciens dominions. La France a retrouvé en 1918 un réseau d'Alsace-Lorraine équipé en signalisation allemande dont il subsiste quatre-vingts ans plus tard de nombreux témoignages. Les territoires de l'ancien empire austro-hongrois ont connu une variante du système d'Europe centrale qui n'a pas résisté aux éclatements et au passage à la signalisation lumineuse. En un siècle l'homogénéité créée quelque part peut avoir engendré la diversité ailleurs, et inversement. 
Les organismes de normalisation recherchent l'harmonisation et l'unification sur des bases communes. Mais lesquelles? Ils n'ont pas toujours eu un poids suffisant sur un sujet qui n'a pas été jugé prioritaire, au contraire de ce qui s'est passé pour la route. Parfois se sont dissimulés dans cette recherche des enjeux industriels ou politico-économiques. L'exemple récent est celui de l'OSJD (organisation des chemins de fer des pays de l'Est) qui, vers la fin des années cinquante, a fait adopter dans la plupart des pays membres des principes communs de signalisation lumineuse ; il est vrai que sa tâche était facilitée par l'appartenance de ces pays à la signalisation dite d'Europe centrale.

L'obsolescence, en éliminant les réalisations anciennes ou techniquement dépassées, a contribué à homogénéiser les signalisations. C’est le cas des héritages des anciens réseaux qui ont subsisté après leur fusion en des réseaux nationaux. L'Allemagne avec la signalisation bavaroise, l'Espagne, la France et la Grande-Bretagne en ont vécu des exemples notoires et durables.

\section{Les trois écoles en matière de signalisation des bifurcations}

La bifurcation est un thème qui se prête bien à une étude comparée des pratiques européennes, car elle rassemble dans un cas simple l'essentiel des besoins en signalisation des voies principales : commander l'arrêt, le passage à vitesse normale ou à vitesse réduite. Elle permet aussi d'illustrer l'évolution vers la signalisation lumineuse et les conséquences de l'adoption d'aiguilles allongées.

\section{Les signaux d'arrêt et de passage à vitesse réduite}

Bien que née comme le rail en Angleterre, la signalisation ne s'est pas développée dans l'homogénéité qu'on pouvait espérer. Des divergences précoces allaient en effet déboucher de façon quasi - irréversible sur trois « écoles». Pour les illustrer nous avons retenu le cas d'une bifurcation comportant deux branches déviées et une branche directe (planche II) afin de montrer les principes mis en œuvre et les principales évolutions, telles l'adoption du feu vert de voie libre et de la couleur jaune des signaux d'avertissement et de marche prudente ${ }^{1}$.

L'école anglaise utilise un signal à bras multiples placés côte à côte ou superposés ; ces bras sont rangés dans l'ordre des directions, pris de la gauche vers la droite, seul celui qui correspond à la direction donnée est ouvert. Le même signal cumule trois fonctions :

1- Les deux changements de couleur sont intervenus en même temps en France en 1936 (code Verlant) ; ils sont intervenus plus tôt et séparément en Allemagne et en Grande-Bretagne. 

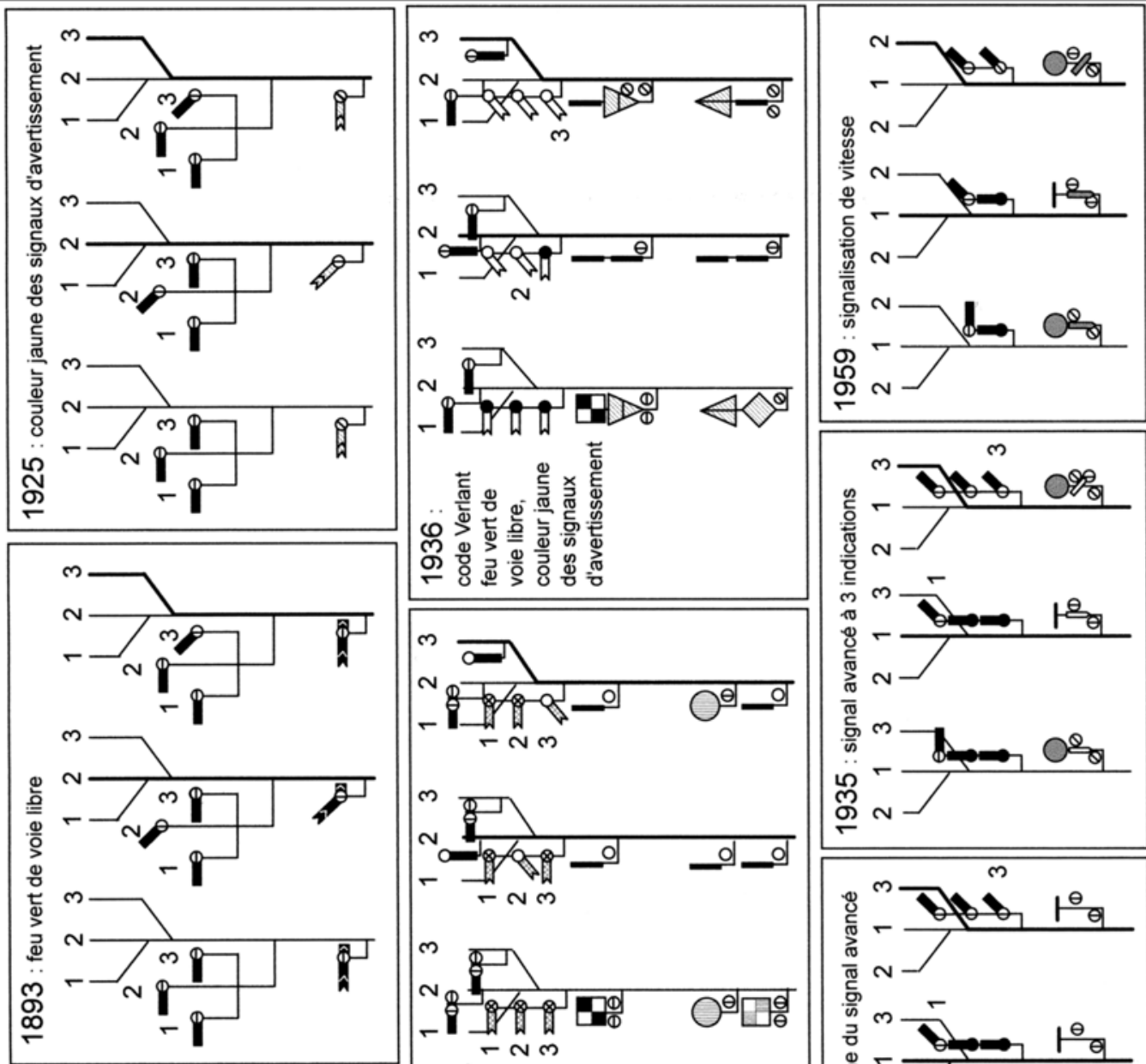

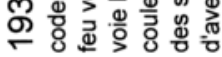
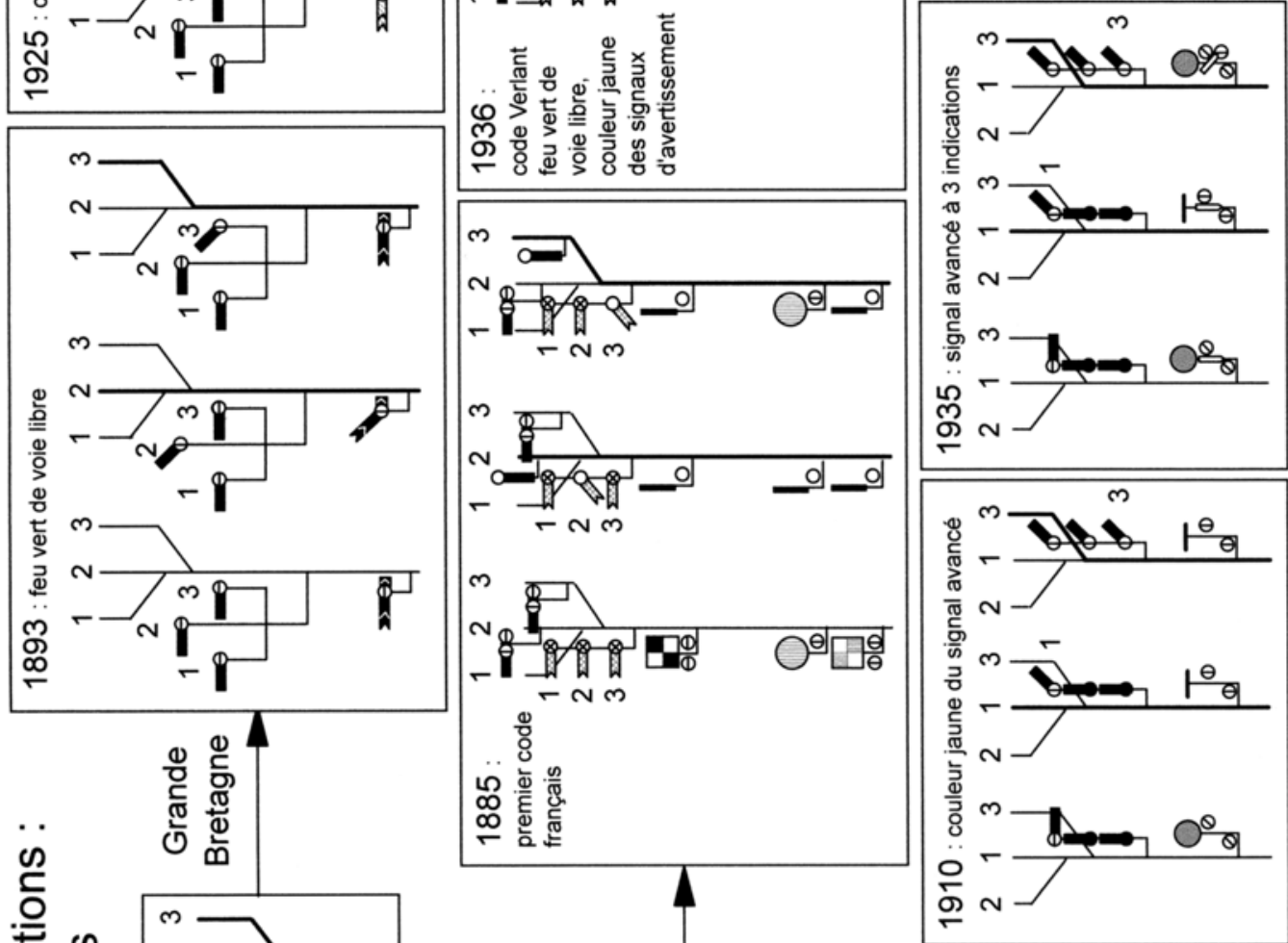

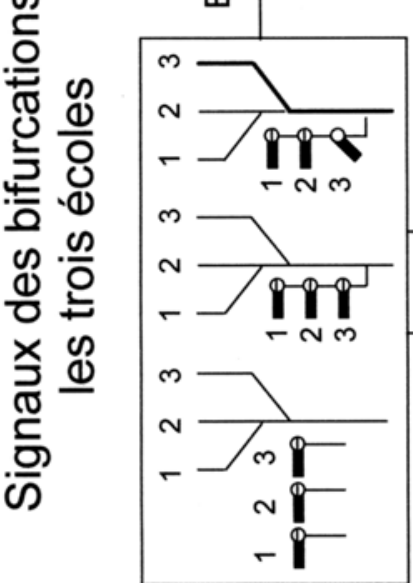
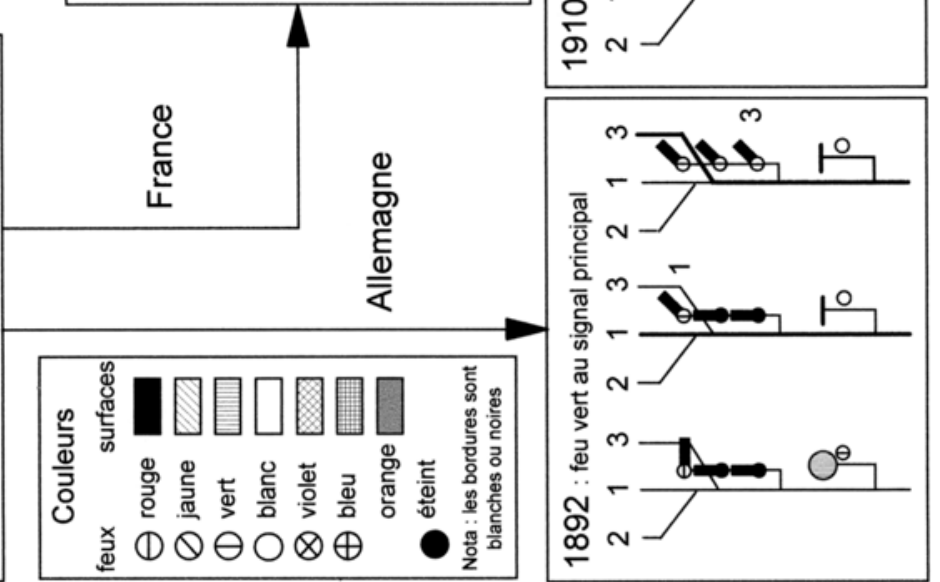

๑๑ Daniel Wurmser 2002 
- commander l'arrêt s'il faut interdire le passage sur les aiguilles, la bonne,

- indiquer la direction pour que le mécanicien s'assure qu'elle est

- espacer les trains, chaque bras n'étant ouvert que si la direction correspondante est libre.

Sur les voies principales apparaissent par la suite des « chandeliers » à bras juxtaposés où le bras relatif à la branche directe est plus haut que les autres. Le principe en est repris avec de légères différences en Belgique, Irlande, Italie et aux Pays-Bas (planche III), et des différences plus marquées au Danemark. Mais Allemands et Français objectent que :

- le passage est autorisé par un signal à voie libre au milieu d'autres à l'arrêt, ou de nuit par un feu de voie libre parmi des feux rouges d'arrêt, d'où difficulté de lecture et franchissement de feux rouges ;

- aucune indication de vitesse n'est donnée et, même si elle fait partie de la connaissance de son itinéraire par le mécanicien, aucun rappel n'en est fait sur le terrain.

L'école française. Le premier code français unifié, celui de 1885, répond aux objections précitées en protégeant les aiguilles par un unique signal d'arrêt absolu, le carré à damier rouge et blanc, qui est ouvert quelle que soit la direction donnée. Il est complété le cas échéant par un disque vert qui indique à distance si le passage implique un ralentissement, et par un indicateur de direction, vestige du système britannique (importé par le PLM) dont la couleur violette, peu éclatante, dénote le caractère accessoire. La France a donc opté dès 1885 pour une signalisation de vitesse. Ce code renonce aux combinaisons de bras en faveur ailleurs et affecte à chaque indication majeure ou chaque fonction son signal (et, à partir de 1936, sa silhouette) ; le signal à cocarde prédomine bien que les tenants du sémaphore lui reprochent son invisibilité à voie libre ${ }^{2}$. Un damier vert et blanc annonce à distance la position du ou des signaux d'arrêt. Trois autres signaux d'arrêt sont prévus dont seul le premier joue un rôle dans notre exemple : le sémaphore pour espacer les trains (un par branche de la bifurcation), le carré ou disque jaune d'arrêt absolu pour les voies de service, et un signal d'arrêt différé, le disque rouge ${ }^{3}$, à la fois signal d'avertissement et signal d'exécution. Le code «Verlant» de

2- Le Portugal présente une palette verticale à damier vert et blanc quand la cocarde esteffacée.

3- Le disque rouge compense sa complexité réglementaire par l'économie d'un signal. Il commande la marche à vue, puis l'arrêt au premier obstacle (train, signal même ouvert, aiguille). En France il poursuit sa carrière sous forme lumineuse bien que promis à la disparition depuis des lustres, alors que l'Espagne et le Portugal l'éliminent systématiquement. 


\section{Exemples de chandeliers de type britannique}

sans annonce à distance de la direction déviée (avertissement maintenu fermé)
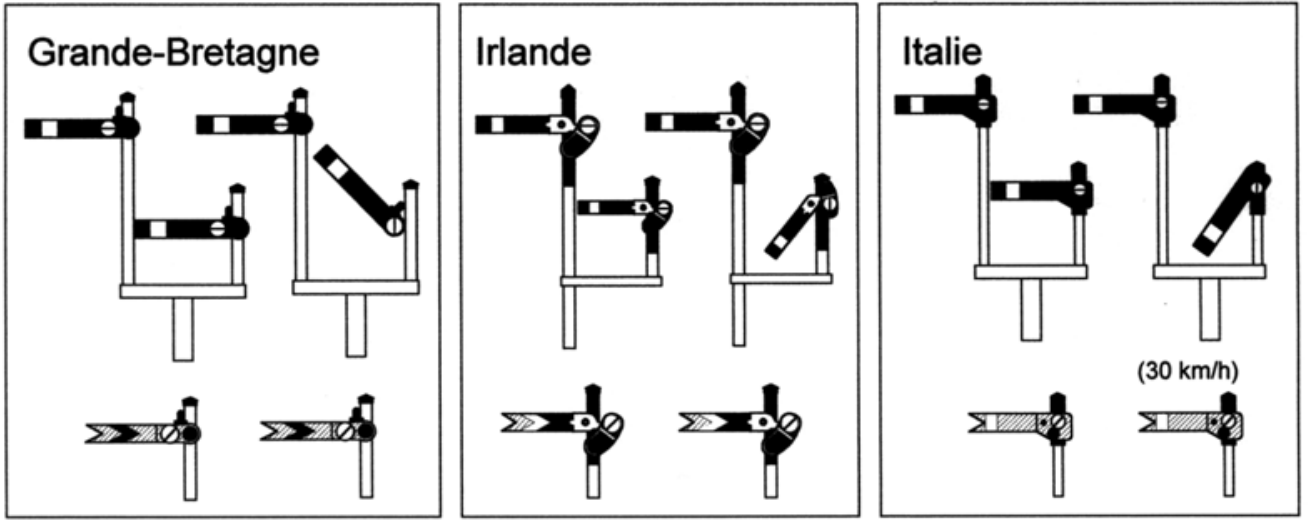

Belgique (système à 2 positions)

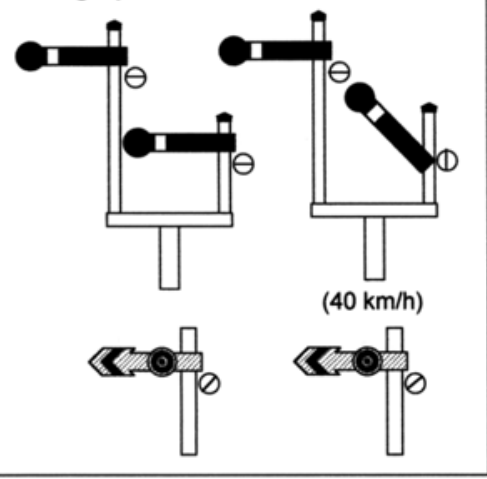

avec annonce à distance de la direction déviée (avertissement à 3 indications)

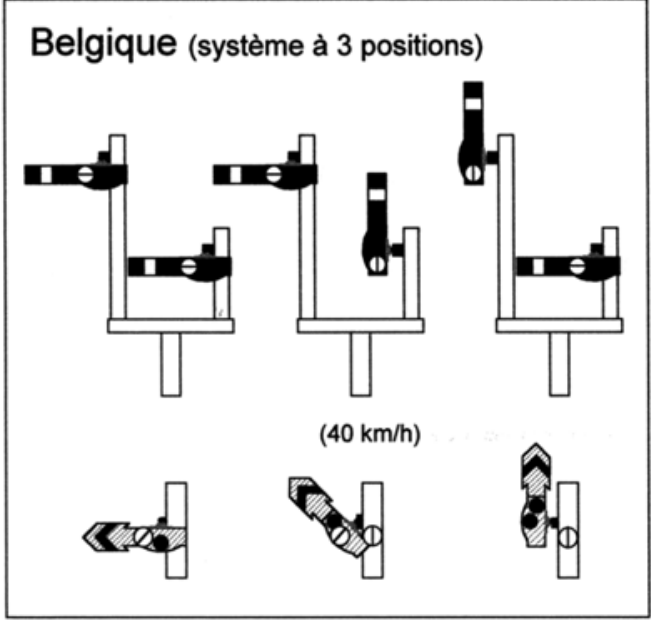

Pays-Bas

Planche III
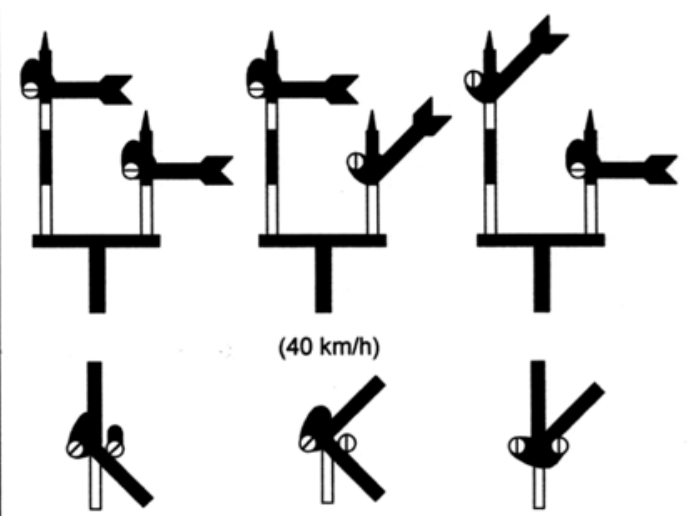

๑ Daniel Wurmser 2002 
1936 reconduit les mêmes principes mais marque l'adoption du feu vert de voie libre et de la couleur jaune pour les signaux d'avertissement et de ralentissement. L'Espagne et le Portugal suivent des principes voisins (planche IV).

L'école allemande recourt à un signal principal (Hauptsignal) à bras superposés. A l'arrêt seul le bras supérieur prend la position horizontale d'arrêt et donne l'unique feu rouge ; les autres bras sont relevés le long du mât, donc " effacés ", et ne donnent pas de feu. Le bras du haut est relevé à $45^{\circ}$ et le feu rouge est remplacé par le feu de voie libre pour autoriser le passage, cependant que :

- vers la branche directe, les autres bras restent effacés et, de nuit, on a un seul feu de voie libre;

- vers la branche déviée, ce sont un, deux ou trois bras qui s'inclinent à $45^{\circ}$ en plus du bras supérieur, donnant chacun le feu de voie libre.

Le lien anglais $« \mathrm{n}^{\mathrm{e}}$ bras $=\mathrm{n}^{\mathrm{e}}$ direction $»$ est rompu, les branches déviées se décomposant en $1^{\text {re }}$ branche déviée (deux ailes à $45^{\circ}$ ), $2^{\mathrm{e}}$ branche déviée (trois ailes à $45^{\circ}$ ). C'est une signalisation d'itinéraire où seul le livret de marche des trains donne la vitesse à respecter sur chaque branche, précision d'autant plus nécessaire que le signal avancé (Vorsignal) qui précède le signal principal est mis à voie libre pour la branche directe comme pour une branche déviée. Par ailleurs l'extinction du feu associé à l'un des bras peut modifier l'indication de direction, ou déformer l'indication de passage sur branche déviée en indication de passage sur la branche directe ${ }^{4}$.

Cette signalisation, la plus utilisée en Europe et en Scandinavie, a reçu le nom générique de signalisation d'Europe centrale (planche V); elle se remarque par ses bras terminés par un rond et la forme circulaire des signaux d'avertissement. La version austro-hongroise s'en distingue par des signaux à claire-voie : ailes rectangulaires des signaux d'arrêt, signal avancé rectangulaire, signal de manœuvre à losange bleu.

Si la Grande-Bretagne est restée fidèle à la règle consistant à ne pas associer de limitation de vitesse aux bras inférieurs des chandeliers, la Belgique et les Pays-Bas leur ont attribué la vitesse de $40 \mathrm{~km} / \mathrm{h}$ et l'Italie de $30 \mathrm{~km} / \mathrm{h}$, ralliant ainsi le camp des partisans de la signalisation de vitesse.

4- C'est pourquoi la Norvège a inversé les indications en adoptant la signalisation de vitesse avec deux ailes à $45^{\circ}$ et deux feux verts pour la branche directe, et une aile à $45^{\circ}$ et un feu vert pour la branche déviée. 


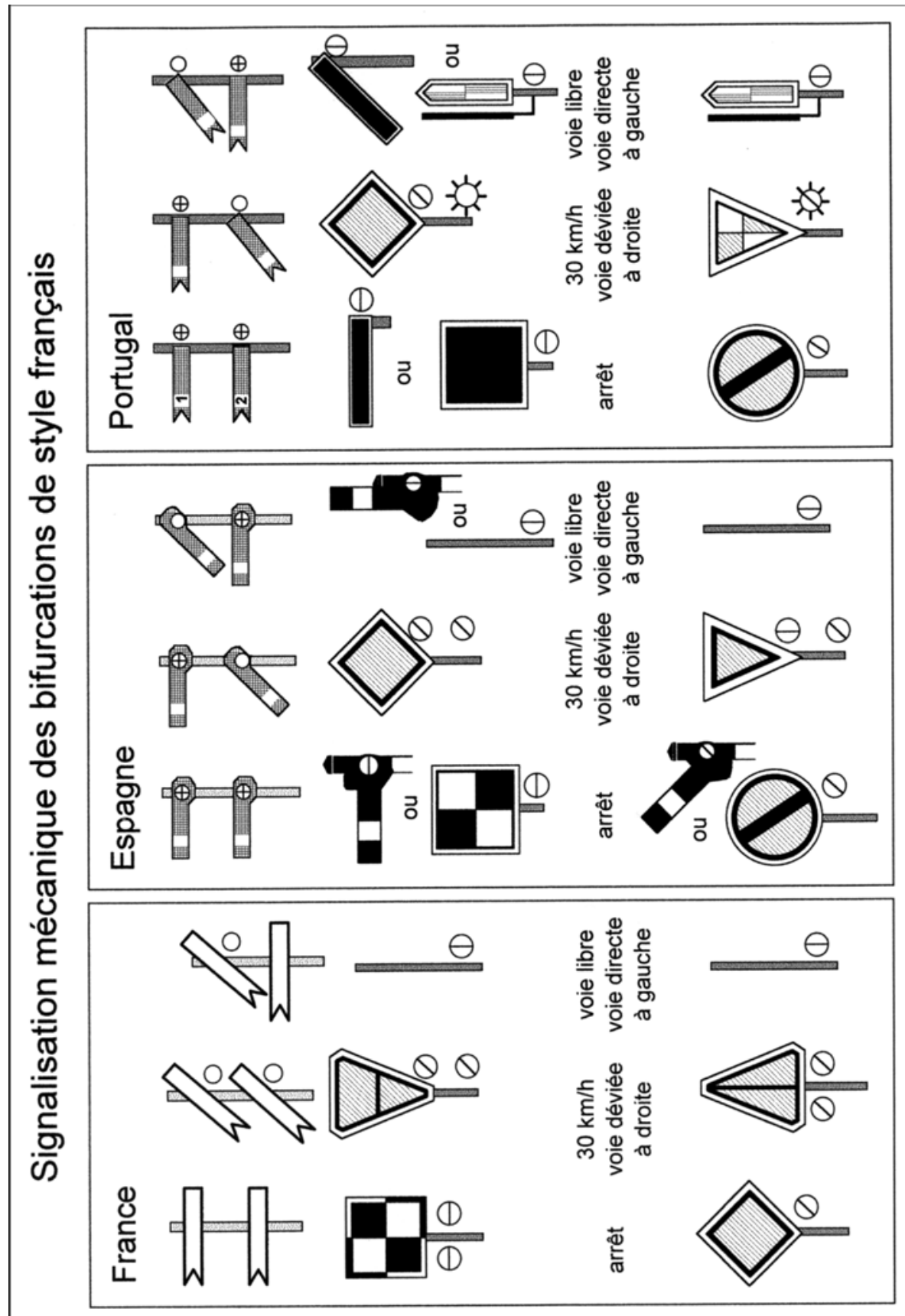

Planche IV

๑ Daniel Wurmser 2002 


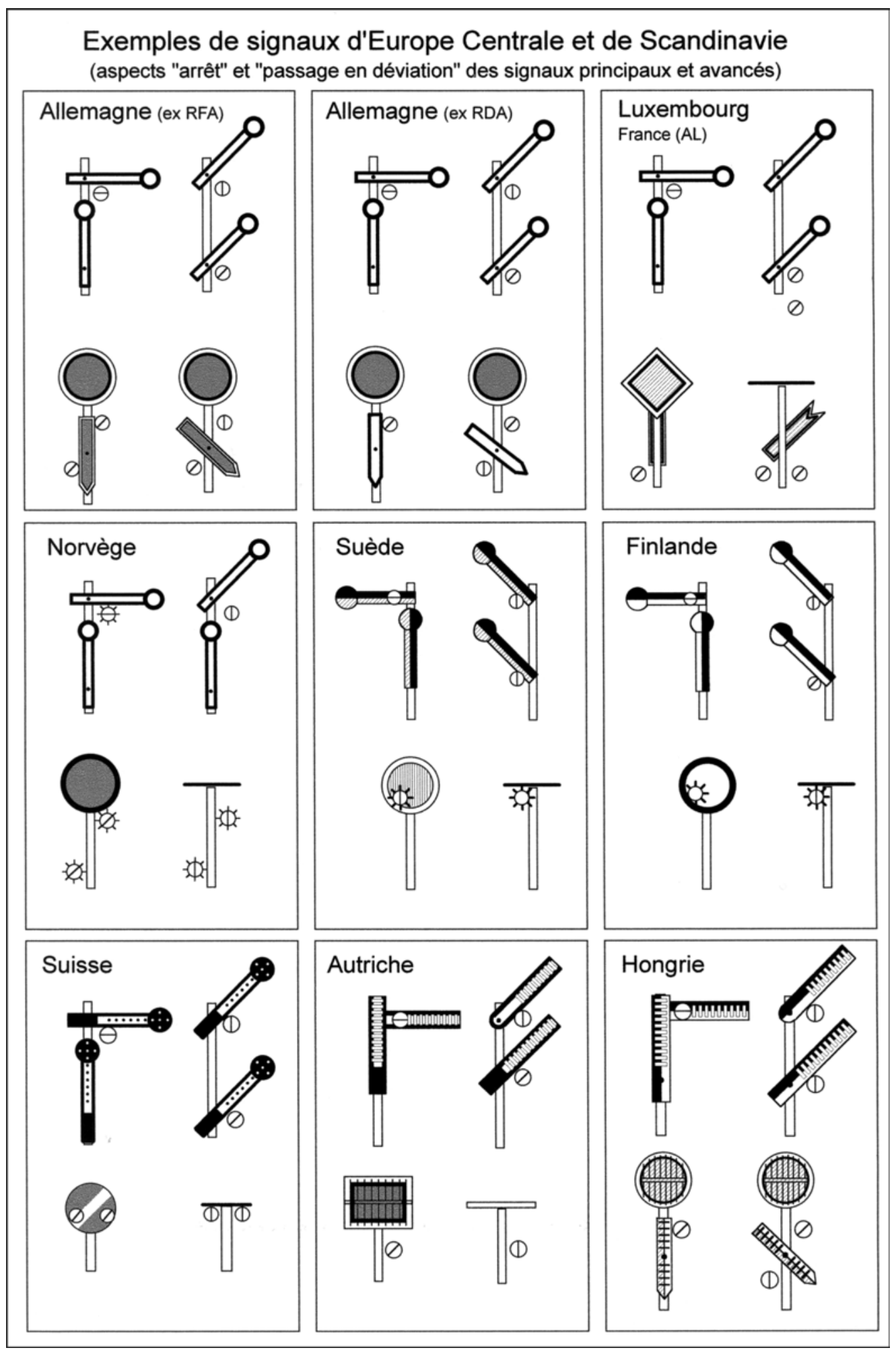


A côté de ces différences, les écoles britannique et allemande convergent sur l'emploi du même signal d'arrêt absolu pour protéger les aiguilles et espacer les trains. Elles ne font pas la distinction française entre carré et sémaphore ; le block est donc absolu et les pénétrations en canton occupé sont interdites, sauf circonstances exceptionnelles. Mais pour compliquer les choses le signal d'arrêt absolu allemand ne s'adresse qu'aux trains et peut être franchi fermé par les manœuvres ; ce n'est le cas ni en Grande-Bretagne, ni en France.

\section{Les signaux d'avertissement}

L'élévation des vitesses a fait naitre le besoin d'un signal d'avertissement (appelé aussi annonciateur ou signal avancé) prévenant le mécanicien qu'il approche d'un signal d'exécution commandant l'arrêt ou le passage à vitesse réduite. La distance qui les sépare fait intervenir la vitesse admise en ligne, les déclivités et les caractéristiques de freinage des trains.

Les écoles française et allemande, pour une fois d'accord en choisissant la cocarde, ont donné à leurs signaux d'avertissement la couleur verte, puis jaune ou orange. L'Angleterre et son école ont adopté un bras sémaphorique différent du bras d'arrêt; de rouge il est devenu jaune, sauf au Danemark, en Irlande et aux Pays-Bas où la couleur rouge a été conservée (bien que donnant un feu jaune). La nuit, les réseaux anglais et français font donner au signal d'avertissement ouvert le même feu vert de voie libre que le signal d'arrêt, mais l'Allemagne ou la Suisse lui font donner deux feux verts pour le distinguer du signal principal à voie libre. Le Danemark, la Finlande, la Norvège et la Suède les repèrent par des feux clignotants jaunes, blancs ou verts.

Aux bifurcations les installations d'origine révèlent les insuffisances suivantes :

- l'Allemagne met à voie libre le signal d'avertissement dès l'instant où le signal qui protège la bifurcation n'est pas à l'arrêt, sans différencier la branche déviée de la branche directe ;

- l'Angleterre, au contraire, maintient fermé le signal d'avertissement si la branche déviée est donnée et ne l'efface que pour la branche directe.

Dans le premier cas, un mécanicien, habitué à être reçu sur la branche directe, a-t-il pu ralentir s'il est reçu sur la branche déviée sans avoir été prévenu ? Dans le second, un mécanicien habituellement reçu sur voie déviée, s'arrêtera-t-il à temps si le signal est à l'arrêt ? Dans les deux cas, la solution a été apportée par des signaux d'avertissement à trois indications : annonce d'arrêt, annonce de passage en déviation ou 
à vitesse réduite, annonce de passage par la branche directe ou sans ralentissement. L'Allemagne et plusieurs pays d'Europe centrale l'ont fait en munissant la cocarde d'avertissement d'un bras additionnel, tandis que d'autres ne l'ont fait qu'en signalisation lumineuse. Dans l'école britannique seuls les Pays-Bas, avec un signal d'avertissement à deux bras, et la Belgique en 1919 ont créé les aspects correspondants (planche III en bas). L'Italie le fera en signalisation lumineuse. La Grande-Bretagne ne généralisera pas une solution qu'elle a pourtant utilisée à petite échelle, avec un chandelier d'avertissements à bras jaunes répétant la position des bras rouges.

\section{Le passage à la signalisation lumineuse}

Nous ne reviendrons pas sur les avantages des signaux lumineux : mêmes aspects de jour et de nuit, frabilité, simplicité de commande et d'entretien. Toutefois des précautions doivent être prises pour qu'une extinction accidentelle ne fasse pas apparaitre une indication moins restrictive. Elles vont de l'allumage d'un feu rouge de secours, au dédoublement des feux (avertissements lumineux autrichiens ou belges donnant deux feux jaunes au lieu d'un en signalisation mécanique), en passant par l'apparition d'une indication restrictive sur le panneau en dérangement (en Grande-Bretagne l'extinction d'un des deux feux jaunes du signal de pré-avertissement le transforme de facto en signal d'avertissement) ou sur le panneau qui le précède (France). Il faut indiquer enfin si le panneau éteint peut présenter l'indication d'arrêt et, si c'est le cas, s'il peut être franchi à l'issue d'un délai ou après autorisation délivrée par un agent sédentaire (aiguilleur, chef de circulation, régulateur).

La règle la plus communément admise pour les signaux lumineux a été de présenter les mêmes feux que leurs homologues mécaniques. En Allemagne, en Autriche ou en Suisse, les signaux lumineux sont la réplique des signaux mécaniques : par exemple quand un signal d'arrêt est combiné à un signal d'avertissement, chaque signal donne ses feux et, s'ils sont tous les deux à voie libre, ils donnent chacun cette indication, en l'occurrence un feu vert et deux feux verts.

Les planches VI, VII et VIII illustrent l'équivalence entre signalisation mécanique et signalisation lumineuse dans le cas du passage des aiguilles par la branche déviée. Si les écoles française et d'Europe centrale montrent la continuité des aspects, on ne peut en dire autant de l'école britannique où les chandeliers, après avoir été remplacés par des chandeliers lumineux à panneaux et à feux rouges multiples, ont cédé la place à autant de types de signaux à panneau et à feu rouge unique que de pays. On se reportera pour cela aux aspects présentés en Belgique, Grande-Bretagne, Italie et aux Pays-Bas. La Grande-Bretagne et l'Irlande 


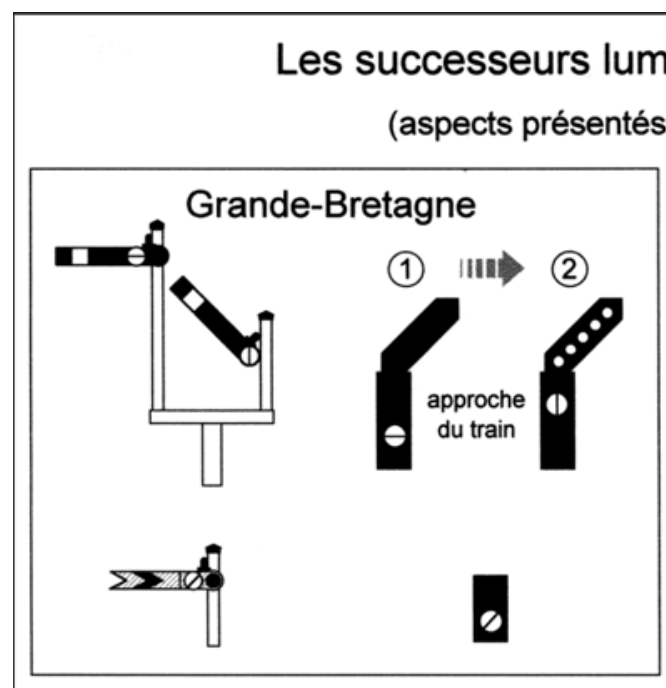

\section{Les successeurs lumineux des chandeliers}

(aspects présentés pour la voie déviée)
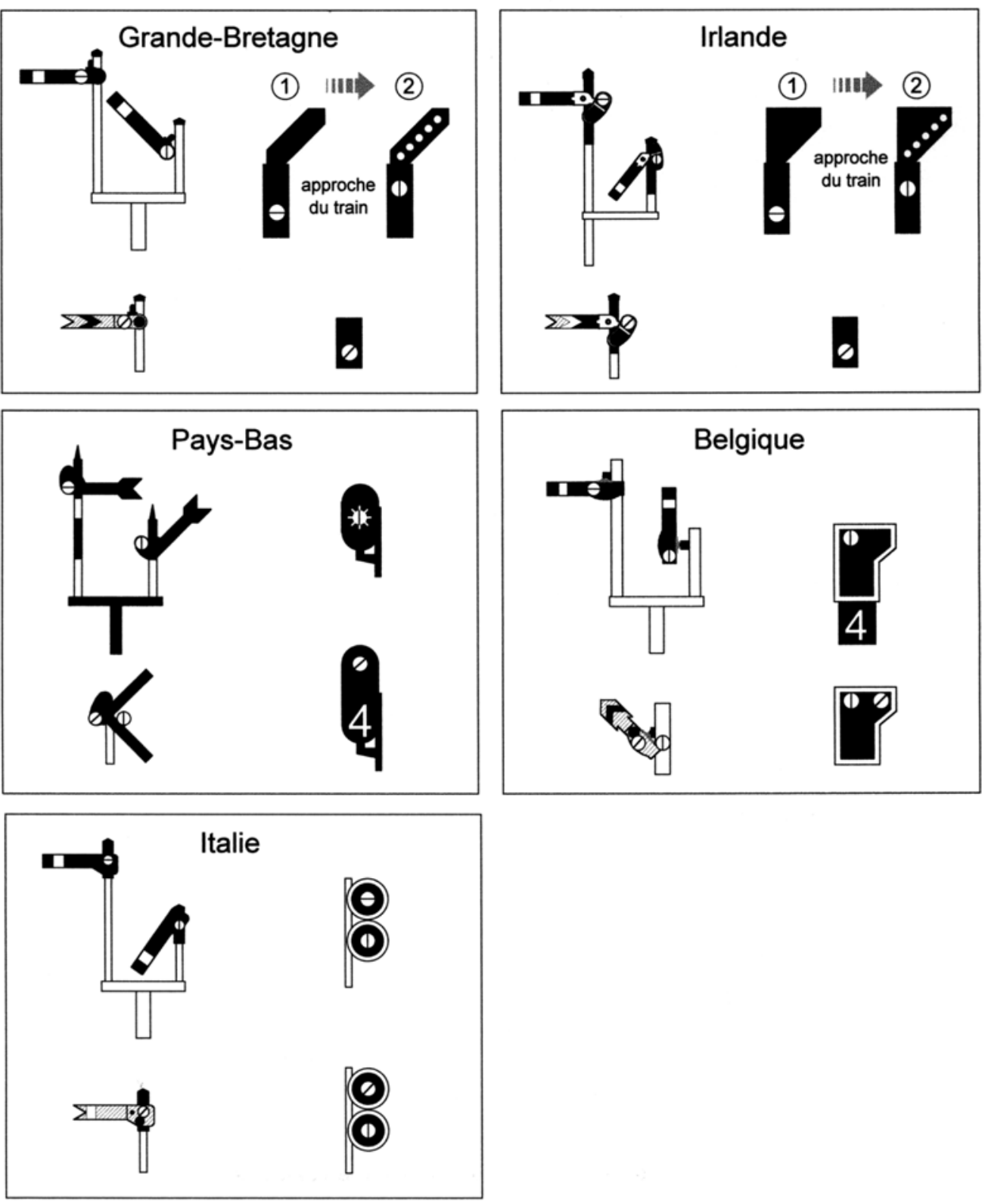

Planche VI

๑ D Daniel Wurmser 2002 
Signalisation du passage à $30 \mathrm{~km} / \mathrm{h}$ sur aiguilles en déviation en Espagne et en France

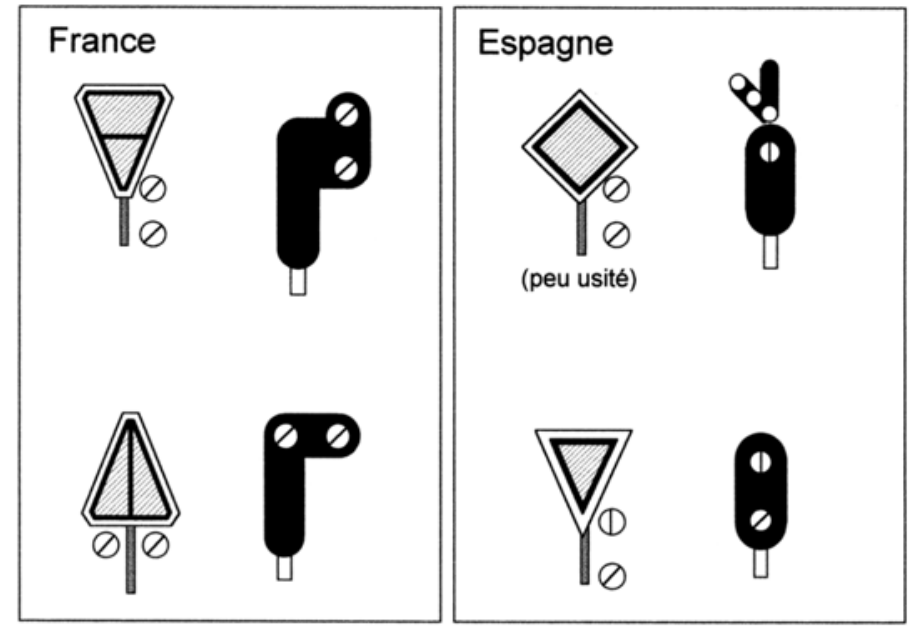

Différenciation des vitesses sur aiguilles en déviation

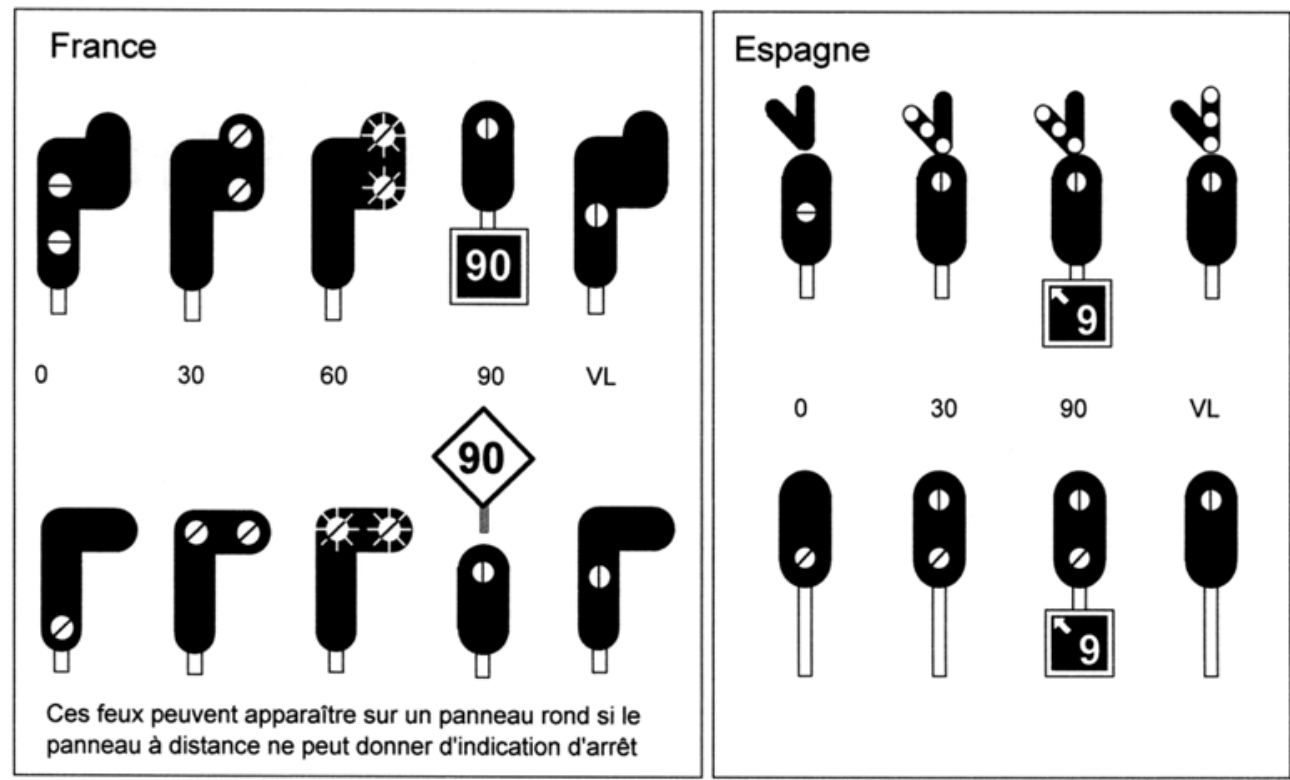

Planche VII

๑ Daniel Wurmser 2002 


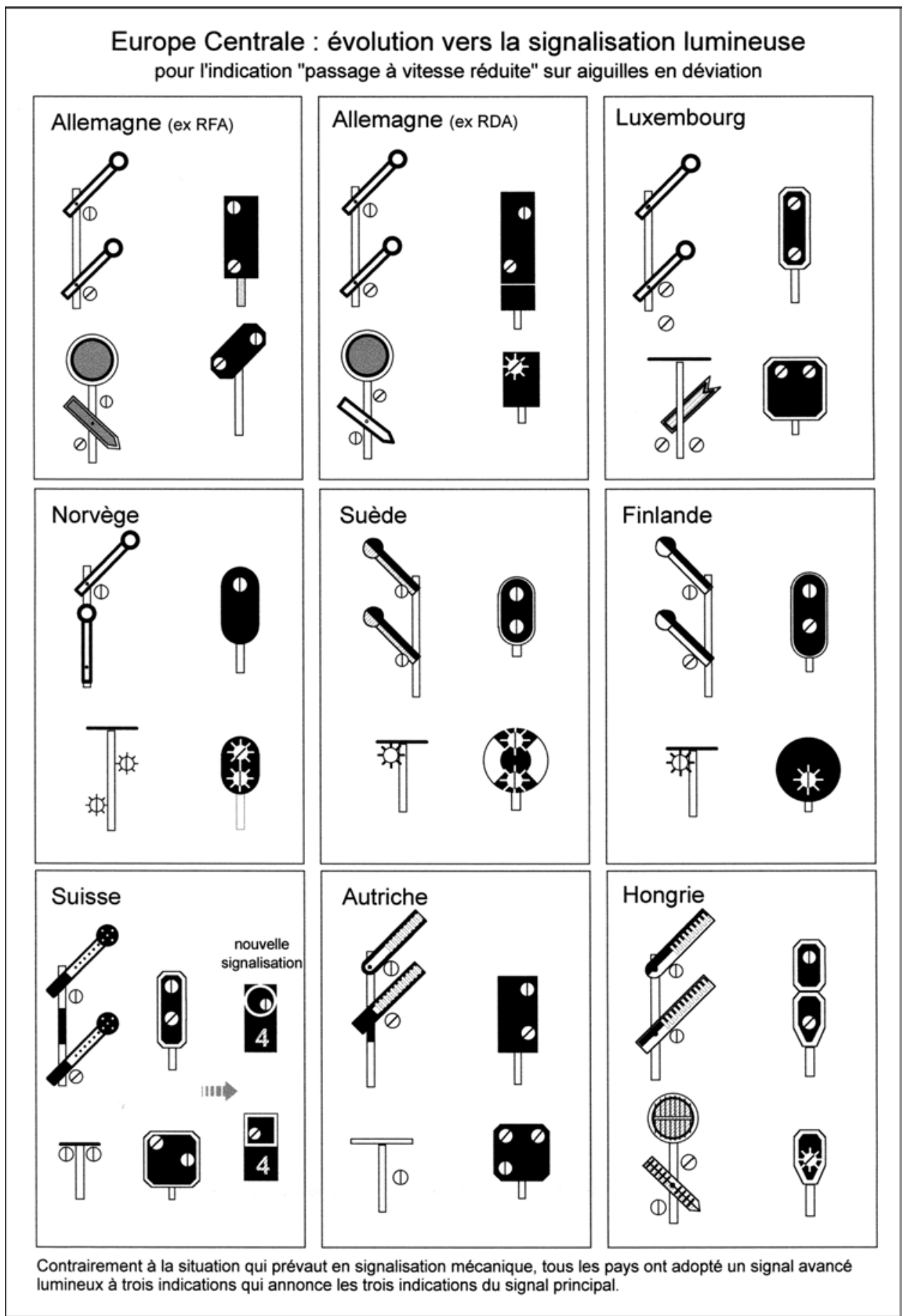

Planche VIII

๑ Daniel Wurmser 2002 
sont restées fidèles à une signalisation de direction mais avec contrôle d'approche. Cette technique consiste à ne pas ouvrir les signaux d'arrêt et d'avertissement protégeant une aiguille en déviation dès la préparation de l'itinéraire. Seul le signal d'arrêt s'ouvre lorsque le train a atteint un point situé après son avertissement et a donc suffisamment ralenti ; en même temps s'allume l'indicateur de branche déviée formé d'une ligne inclinée de feux blancs lunaires ${ }^{5}$. Il va de soi que le conducteur doit se tenir prêt au cas où le signal d'arrêt ne s'ouvrirait pas.

\section{Aiguilles longues et signalisation de vitesse}

Pour les pays qui ont choisi la signalisation de vitesse, c'est-à-dire maintenant presque tous, les aiguilles longues ont imposé la création d'indications de vitesses souvent supérieures à la vitesse basse « traditionnelle » $(30 \mathrm{~km} / \mathrm{h}$ en Espagne, en France et en Italie, $40 \mathrm{~km} / \mathrm{h}$ en Allemagne et en Europe centrale). Le plus souvent les signaux correspondants s'ajoutent à l'indication de vitesse basse, de telle sorte que leur extinction laisse subsister celle-ci, ce qui joue dans le sens de la sécurité.

Ils revêtent quatre formes (planches IX et X) :

- des chiffres exprimant en clair la vitesse autorisée en $\mathrm{km} / \mathrm{h}$ ou en dizaines de $\mathrm{km} / \mathrm{h}$;

- des feux clignotants dont il existe plusieurs variantes (lent, rapide, simultané, alterné) ;

- des combinaisons de feux jaunes et verts ;

- des traits lumineux ou lignes de petits feux blancs ou colorés.

A la fin des années cinquante, l'OSJD a défini une signalisation commune aux pays de l'Est (planche $\mathrm{X}$ en bas) qui reprend certains principes d'Europe centrale tel l'allumage du feu jaune inférieur du panneau chaque fois qu'une aiguille est prise en déviation (en pointe ou en talon). Deux ou trois paliers de vitesse sont introduits pour les aiguilles longues ; il leur correspond des lignes horizontales de petits feux verts ou jaunes qui s'ajoutent au feu jaune de déviation si la vitesse est supérieure à $40 \mathrm{~km} / \mathrm{h}$. Les feux supérieurs portent les feux d'annonce du signal suivant (jaune fixe s'il est à l'arrêt, vert fixe s'il est à voie libre pour la branche directe, clignotants jaunes ou verts s'il indique le passage à vitesse réduite, avec un clignotement lent et un clignotement rapide). Le système OSJD, qui consomme peu de feux, hiérarchise les feux sur un même signal et supprime le «cumul des feux » de l’Europe centrale

5- S'il y a plus d'une direction déviée, l'indicateur comprend plusieurs lignes de feux blancs disposés en rosace. Aucune ligne n'est allumée pour la branche directe. 


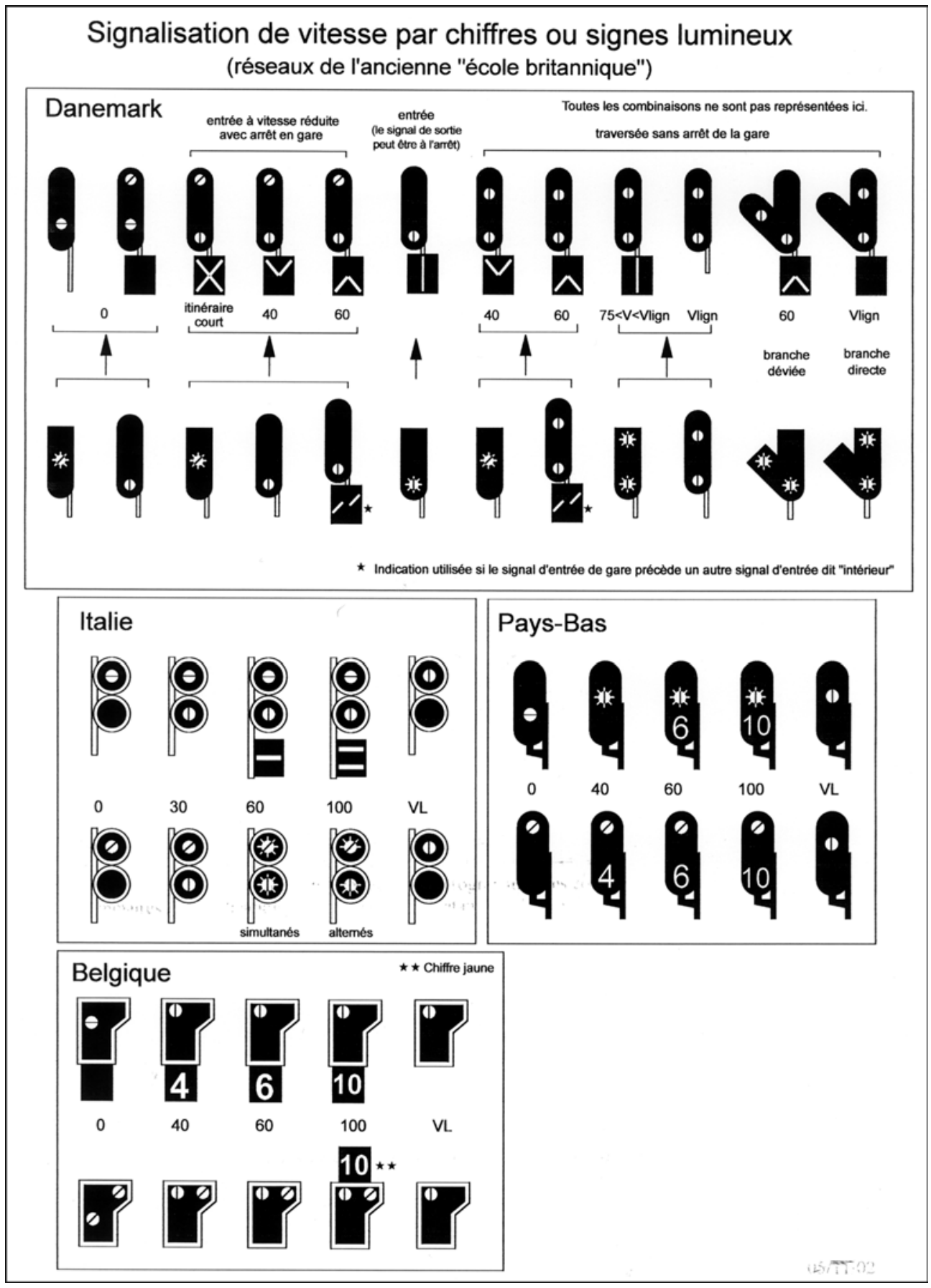

Planche IX

๑ Daniel Wurmser 2002 


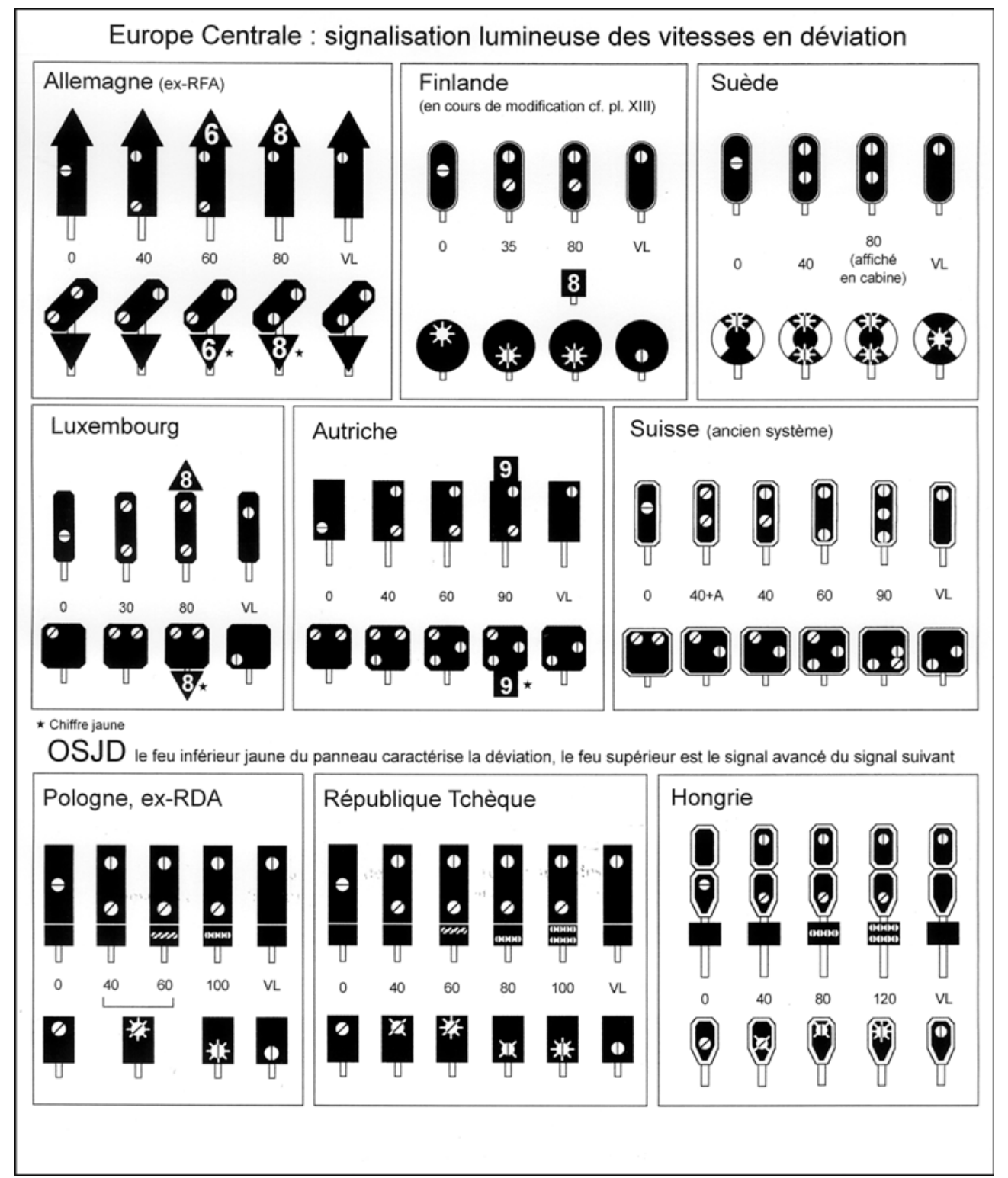

Planche $\mathrm{X}$

๑) Daniel Wurmser 2002 
avec un feu vert unique de voie libre. Le panneau de pleine voie donne ainsi un feu rouge, un feu vert ou un feu jaune comme tant de ses homologues européens.

La Suisse est l'exemple de pays utilisant des combinaisons de feux assez complexes. Par exemple un signal principal autorisant le passage à $90 \mathrm{~km} / \mathrm{h}$ et annonçant le même taux au signal suivant, présente trois feux verts en ligne verticale coiffant deux feux verts et un feu jaune en équerre. C'est pourquoi, conscients d'arriver à une limite, ses chemins de fer mettent en place progressivement une nouvelle signalisation à feux colorés uniques et affichage numérique des vitesses.

\section{Les signaux du block automatique à quatre indications}

Dans les zones où la densité des signaux est élevée, des signaux peuvent être installés entre un signal d'arrêt et son signal d'avertissement entrainant des imbrications de signalisation telles qu'un signal d'avertissement peut annoncer plusieurs signaux d'arrêt, ou plus tard, en raison du principe de la continuité de la signalisation d'avertissement ${ }^{6}$, plusieurs avertissements peuvent annoncer un signal d'arrêt. En signalisation mécanique cela créait des dépendances d'autant plus complexes que des postes différents intervenaient et qu'il en résultait souvent pour le mécanicien des difficultés d'interprétation.

La signalisation lumineuse a contribué à résoudre le problème. Mais une fois de plus l'Europe s'est partagée en deux groupes, «l'Europe centrale » et les autres, c'est-à-dire la Belgique, l'Espagne, la France, la Grande-Bretagne, l'Italie, les Pays-Bas, le Portugal.

L'Europe centrale garantit la distance de freinage entre un signal d'arrêt et son avertissement ; s'il y a d'autres signaux dans l'intervalle, un " répétiteur » assure la continuité de l'indication d'avertissement grâce à un aspect particulier rappelant qu'il est à une distance inférieure à la distance de freinage. Le même principe vaut pour les signaux annonçant le franchissement des aiguilles à vitesse réduite. Par ailleurs, si certains signaux sont des répétiteurs permanents dotés d'un feu blanc ou d'un repère fixes, d'autres peuvent, selon les besoins du moment, être avertissement ou répétiteur. Le répétiteur d’avertissement est souvent obtenu en ajoutant un feu blanc aux feux de l'avertissement.

6- Ce principe vise à se prémunir contre la séquence avertissement, voie libre, arrêt qui peut conduire le mécanicien à croire qu'il peut reprendre sa vitesse au second signal, en lui substituant avertissement, avertissement, arrêt (ou pré-avertissement, avertissement, arrêt). Il faut donc combiner un signal d'avertissement au second signal si c'est un signal d'arrêt. 
Les autres pays posent en principe que le dernier signal précédant un signal d'arrêt est un signal d'avertissement, fût-il à une distance inférieure à la distance de freinage. Si tel est le cas, le respect de la distance de freinage est obtenu en le faisant précéder d'un autre signal d'avertissement, ou d'un signal de pré-avertissement ou préannonce introduisant éventuellement une limitation de vitesse (en France et en Italie un feu jaune clignotant, en Grande-Bretagne deux feux jaunes en ligne verticale). Seule la Belgique a introduit un pré-avertissement mécanique dans ses signaux à trois positions de 1919 avec la palette jaune à 45 (comme pour l'annonce à distance d'une voie déviée).

Pour illustrer le fonctionnement d'un block à quatre indications, les aspects pris par les signaux au fur et à mesure qu'un train s'éloigne sont, dans l'ordre (cf. planche XI) :

- en Europe centrale : arrêt, répétiteur d'avertissement, avertissement, voie libre ;

- ailleurs : arrêt, avertissement, pré-avertissement (ou préannonce), voie libre.

\section{Les manœuvres}

Les manœuvres restent dans la zone d'action d'un poste et vont partout, sur toutes les voies, dans le sens normal comme en sens inverse, sur des voies libres ou partiellement occupées. Elles circulent à vitesse réduite pour pouvoir s'arrêter dans l'espace de voie en vue (marche «à vue ») ; elles doivent gêner le moins possible les trains et être protégées de ceux-ci. Face à ces spécificités, la tentation a souvent été grande d'apporter la réponse la plus économique possible, à commencer par les petites gares où les manœuvres n'ont souvent obéi qu'à des drapeaux, lanternes ou sifflets, sans oublier les taquets d'arrêt de toutes sortes. Quand des signaux ont été reconnus nécessaires, l'Europe s'est partagée en trois systèmes, avec des «métissages » comme au Danemark et aux Pays-Bas où les principes d'Europe centrale côtoient une signalisation plutôt britannique (cf. planche XII).

En signalisation mécanique les trois systèmes se distinguent par la présence, sur chaque voie :

- de deux signaux d'arrêt : un pour les trains, qui est ignoré des manœuvres, et un pour les manœuvres, qui est ignoré des trains, afin d'autoriser l'un ou l'autre de ces mouvements ;

- d'un signal d'arrêt auquel obéissent trains et manœuvres, complété par un signal subsidiaire (petit bras ou disque) qui en autorise le franchissement en manœuvre ou en marche à vue ; 


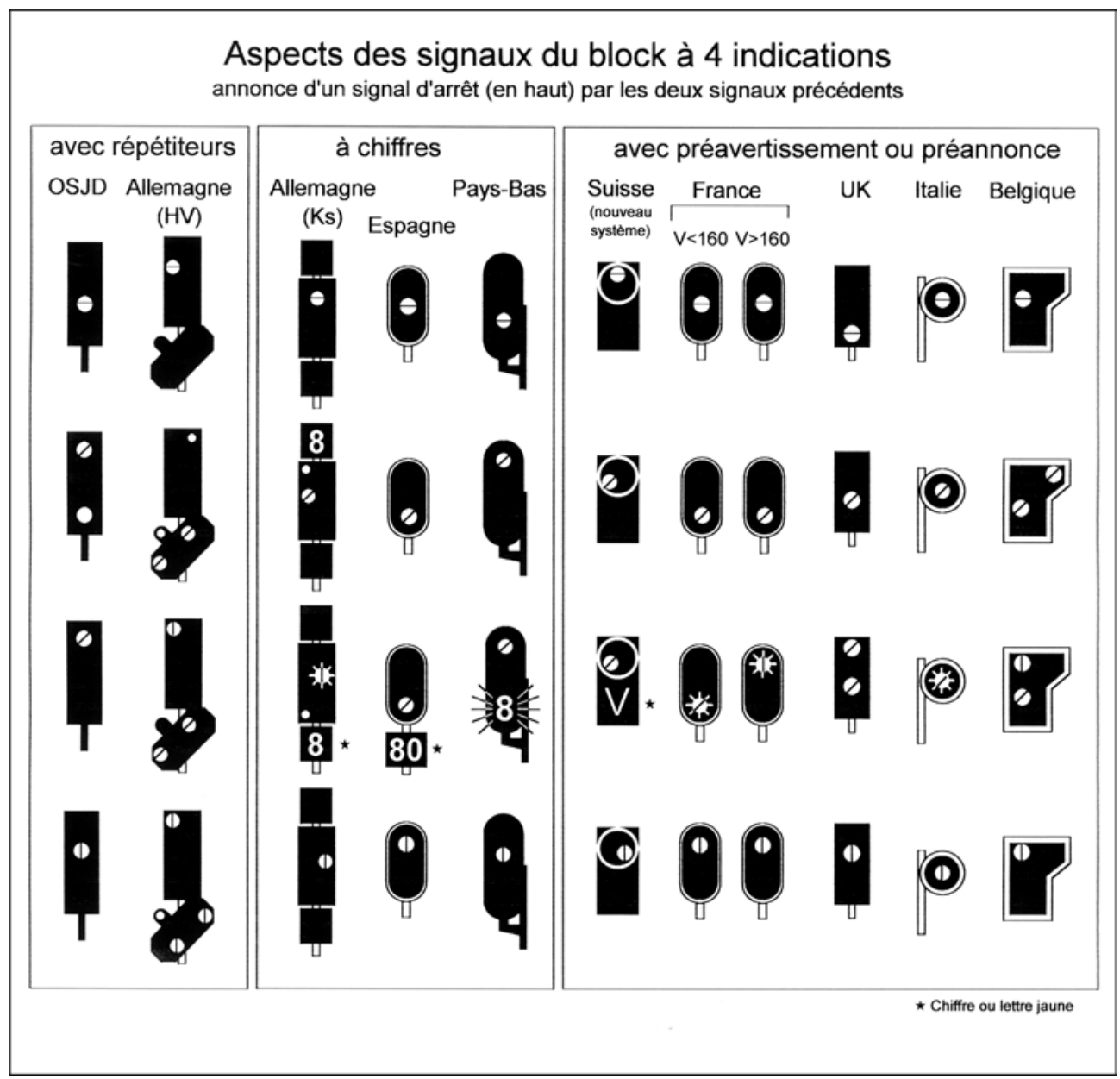

๑ Daniel Wurmser 2002 


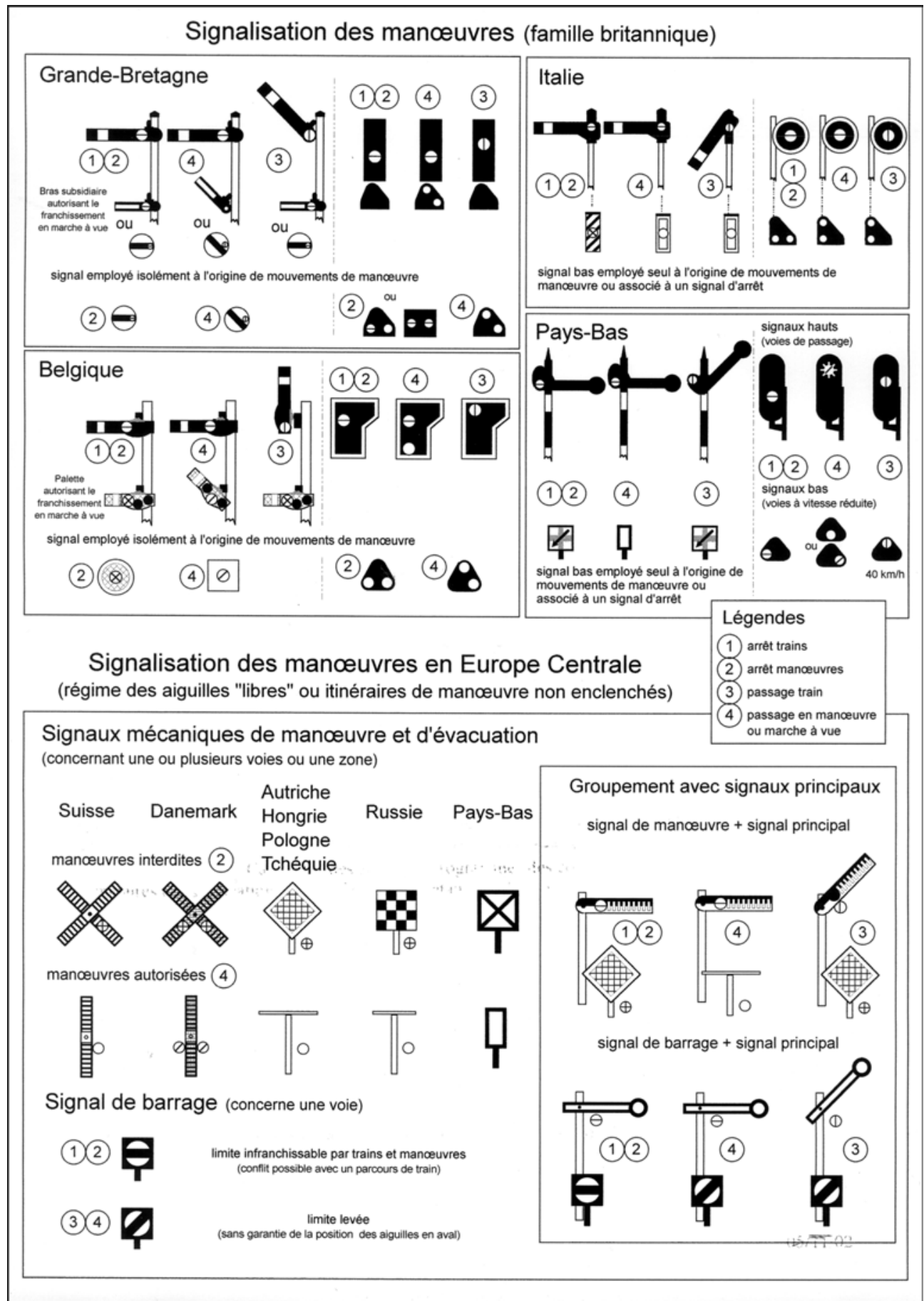

๑ Daniel Wurmser 2002 
- d'un seul signal d'arrêt absolu qui est rouge si l'on est sur une voie principale, violet si l'on est sur une voie de service, auxquels obéissent sans distinction les trains et les manœuvres.

Le premier système qui est celui d'Europe centrale suppose une distinction réglementaire très nette entre trains et manœuvres; en revanche le second, employé en Grande-Bretagne et le troisième, en France, ne reposent pas sur une séparation aussi marquée et ils assurent par des enclenchements la protection de tous les mouvements et la vérification des positions d'aiguilles.

De manière plus précise, les "manœuvres d'Europe centrale » peuvent franchir fermés les signaux principaux; de ce fait rien n'interdit de mouvoir les lames d'une aiguille occupée par une manœuvre et seule la vigilance de l'aiguilleur et des dirigeants de manœuvre assure la protection des manœuvres entre elles. Cette « liberté » impose de protéger les trains contre l'irruption des manœuvres par:

- des signaux d'interdiction de mancuvres et d'évacuation concernant une voie ou une zone que doit emprunter un train : «ciseaux » suisses, danois, alsaciens, signal de repos bavarois, signaux de voie barrée ${ }^{7}$, signaux de manœuvre austro-hongrois à losange bleu ;

- la mise en position protégeante des aiguilles pour « écarter» les manœuvres du parcours d'un train ;

- linterdiction du talonnage des aiguilles d'où l'importance des lanternes qui aident à en discerner la position, une aiguille non préparée pour la branche d'où on vient laissant supposer qu'elle l'est pour un mouvement arrivant par l'autre branche.

Les lacunes de ce régime ont conduit à son abandon après la Seconde Guerre mondiale dans les postes tout-relais à parcours de manœuvre enclenchés. L'établissement d'un parcours de manœuvre suppose qu'aucun parcours existant de train ou de manœuvre n'entre en conflit avec lui et que les aiguilles sont dégagées ${ }^{8}$. L'établissement d'un parcours de train repose sur la préparation des itinéraires de manouvre qui le composent, suivie de la vérification des conditions relatives à la circulation des trains (dégagement des voies et des cantons, branche

7- Le signal de barrage ou de «voie barrée » allemand (Gleissperrsignal), utilisé dans de nombreux pays et autrefois en Alsace-Lorraine, marque le point que manœuvres et trains ne peuvent dépasser. La préparation d'un parcours de train peut imposer sa fermeture. Ouvert, il indique que le barrage est levé et il n'invite pas à avancer, sauf si le mât porte un repère spécial ; il n'enclenche pas les aiguilles.

8- Le signal de manœuvre de la Suisse et des pays scandinaves donne les indications d'arrêt, de manœuvre à marche normale, ou en marche prudente s'il précède une section occupée ou un signal à l'arrêt. 
directe ou déviée des aiguilles). L'observateur voit donc s'ouvrir les signaux de manœuvre intermédiaires, puis le signal principal qui commande le départ du train. Ces nouveaux signaux de manœuvre, qui s'adressent aux trains et aux manœuvres, sont souvent placés au ras du sol, ce qui est source d'économies. Les pratiques récentes de l'Europe centrale rejoignent donc celles des autres écoles.

Les signaux à positions de feux blancs (d'origine américaine) sont souvent employés et transgressent beaucoup de frontières ; c'est le cas en Autriche, Belgique, Danemark, Italie, Norvège, Suède et Suisse dont les familles étaient différentes. Une version à feux rouges ou blancs est utilisée en Allemagne, en Finlande, en Grande-Bretagne et en Irlande et, sous une forme un peu différente, en Espagne et au Portugal. La France utilise aussi des carrés violets bas délimitant des itinéraires qui jalonnent et fractionnent des itinéraires de trains. Les pays de l'Est ont adopté les mêmes principes : feu rouge pour interdire trains et manœuvres, feu bleu pour interdire les manœuvres, feu blanc pour les autoriser.

Curieusement, malgré son caractère utilitaire, la signalisation des manœuvres sera peut-être le dernier bastion de la signalisation latérale. C'est le cas sur les lignes à grande vitesse françaises.

\section{Deux modifications en cours : I'unification de la signalisation allemande et la nouvelle signalisation finlandaise}

La réunification allemande a rassemblé des signalisations qui avaient notablement divergé en une quarantaine d'années. La DR s'était attachée à réduire le nombre des feux :

- signaux avancés mécaniques ou lumineux à feu unique, jaune ou vert, au lieu de deux sur la DB;

- signaux lumineux à feu rouge unique pour commander l'arrêt absolu aux trains et aux manœuvres quand la DB en employait deux';

- emploi du code OSJD avec hiérarchisation des feux aux signaux combinés (donc feu vert unique de voie libre), la DB ayant conservé un caractère additif (feux verts multiples de voie libre).

Le nouveau système Ks (Kombinations-System) en fait la synthèse en reprenant les chiffres lumineux des limitations de vitesse de la DB et le nombre réduit des feux de la DR. Le feu rouge vaut pour les trains et les manœuvres, l'avertissement ne donne plus qu'un feu jaune, et la voie libre un feu vert. Le feu vert clignotant est introduit (planche XIII). La notion de répétiteur qui existait dans les deux systèmes est conservée.

9- Déjà la DR n'en employait qu'un, mais elle a dû poser un repère spécial sur le mât des signaux principaux mécaniques pour qui le feu rouge unique est franchissable par les manœuvres. 
Des exemples actuels de modification de la signalisation

La réunification de la signalisation allemande

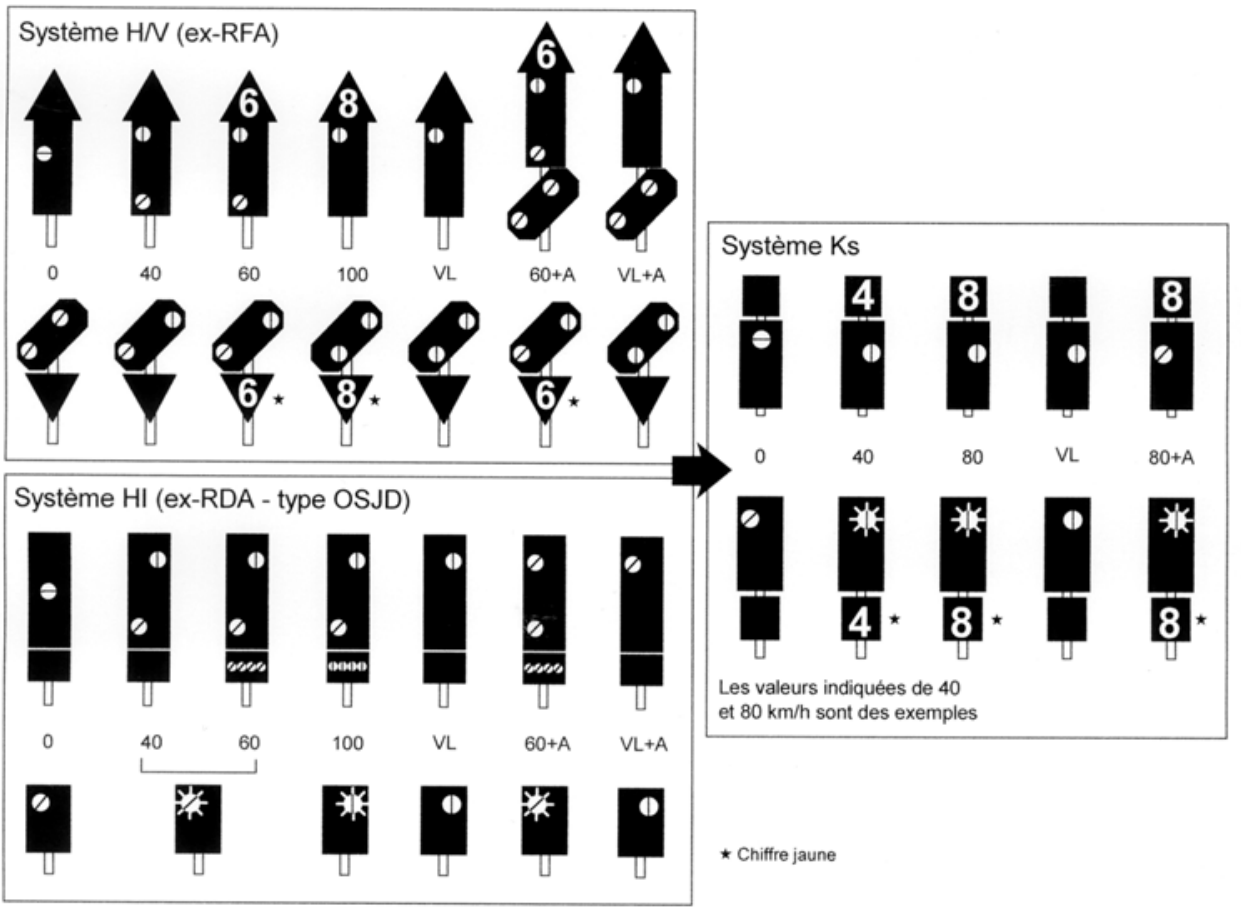

La modification de la sianalisation finlandaise

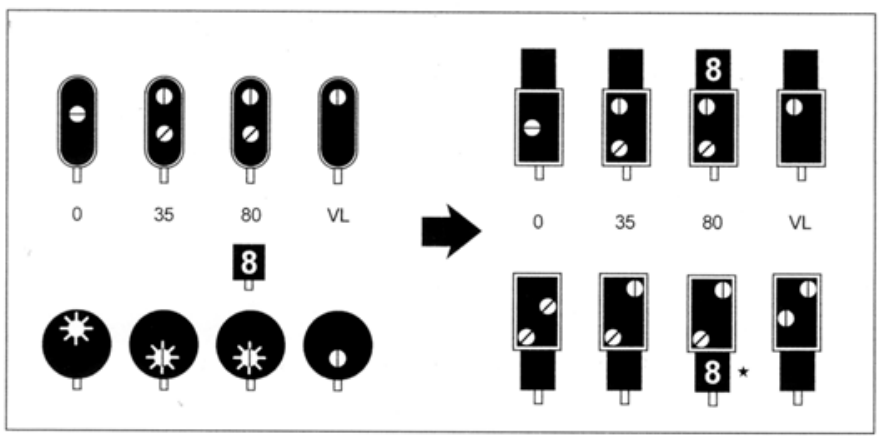

Planche XIII

๑ Daniel Wurmser 2002 
La Finlande qui avait déjà des panneaux de block de pleine voie de forme triangulaire à trois feux (rouge, jaune, vert) change ses signaux principaux des gares et leurs avertissements afin d'éliminer le feu blanc clignotant d'annonce d'arrêt et d'avoir une signalisation plus complète et plus souple des vitesses sur les aiguilles. Les signaux nains de manœuvre font également l'objet de modifications et de simplifications ; ils passent de quatre aspects à trois et donnent deux feux rouges à l'arrêt. On ne peut que noter la parenté avec le système HV de la DB.

\section{Quelques remarques pour conclure}

Aucun système ne s'est avéré parfait et n'a réussi à s'imposer partout. Les divergences ne sont pas récentes et se sont perpétuées. Les fonctions ont plus convergé que les aspects et les réglementations.

Les signaux mécaniques européens vivent probablement leur dernière décennie. On ne cherche plus qu'à en réduire les coûts d'entretien et d'exploitation, en remplaçant l'éclairage par des revêtements réfléchissants bien visibles quand ils sont éclairés par les projecteurs du véhicule de tête.

La recherche d'économies concerne aussi la signalisation lumineuse pour laquelle de nombreux pays se satisfont en gare de signaux placés au ras du sol, surtout pour les manœuvres, cette position étant dans leurs codes significative de circulation à vitesse réduite.

Avec la quasi-généralisation de la signalisation de vitesse se développe l'affichage numérique des vitesses qui est souple et de généralisation facile. Cette technique déborde le cas des aiguilles longues, car plusieurs pays l'emploient en block à cantons courts pour régler les vitesses sur les distances d'arrêt. Elle accroît du même coup la compatibilité avec les techniques informatiques de contrôle de la circulation des trains.

Certains pays, surtout dans le nord de l'Europe, jouent la complémentarité entre signalisation latérale et signalisation en cabine, de manière à élargir la gamme des vitesses sans accroître le nombre des aspects de feux (cf. la Suède, planche X)

Et la normalisation? Il est douteux qu'on puisse user de ce terme, car la situation est encore moins enviable que pour la traction électrique où l'Europe a fini par se ranger sous quatre bannières. Les planches ont apporté le témoignage de cette étonnante diversité. Non seulement des problèmes de masse critique, de coût et de cohérence ont progressivement réduit les probabilités d'unification, mais elle n'a pas été jugée prioritaire. Pour ajouter au tableau une note positive, remarquons que lorsque l'Allemagne et la Suisse auront terminé la mise en place de leur 
nouvelle signalisation, nombreux seront les pays - mais pas tous - pour qui le panneau de block de pleine voie à trois indications présentera le feu rouge d'arrêt, jaune d'avertissement, vert de voie libre.

L'interopérabilité des engins moteurs, ainsi que l'arrivée d'opérateurs ayant leur propre matériel peuvent remettre la question sur le tapis. Déjà des engins hébergent plusieurs systèmes de répétition ou de signalisation en cabine. En outre les technologies évoluent plus vite qu'autrefois ; l'informatique s'approprie de plus en plus de fonctions. L'ETCS (European Train Control System) en est un exemple, mais ce n'est plus notre sujet. 